\title{
1 The Bradyrhizobium sp. LmicA16 type VI secretion system is required for efficient nodulation \\ 2 of Lupinus spp.
}

4 Tighilt $\mathrm{L}^{1,2}$, Boulila $\mathrm{F}^{1}$, De Sousa $\mathrm{BFS}^{2,3}$, Giraud $\mathrm{E}^{4}$, Ruiz-Argüeso $\mathrm{T}^{2,3,6}$, Palacios $\mathrm{JM}^{2,3}$, Imperial J ${ }^{2,5}$, Rey L $\mathrm{L}^{2,3}$

5

61 Laboratoire d'Ecologie Microbienne, Faculté des Sciences de la Nature et de la Vie, Université de Bejaia,

706000 Bejaia, Algeria.

82 Centro de Biotecnología y Genómica de Plantas, Universidad Politécnica de Madrid (UPM)-Instituto

9 Nacional de Investigación y Tecnología Agraria y Alimentaria (INIA), Campus de Montegancedo, 28223

10 Madrid, Spain,

113 Departamento de Biotecnología y Biología Vegetal, ETSI Agronómica, Alimentaria y de Biosistemas,

12 Universidad Politécnica de Madrid 28040 Madrid, Spain

134 IRD, Laboratoire des Symbioses Tropicales et Méditerranéennes (LSTM, UMR $14 \mathrm{IRD} /$ SupAgro/INRA/Université de Montpellier/CIRAD, TA-A82/J-Campus international de Baillarguet, 1534398 Montpellier Cedex 5, France

165 Instituto de Ciencias Agrarias, CSIC, 28006 Madrid, Spain.

176 In memoriam

18

Corresponding author email: luis.rey@upm.es

Lilia Tighilt ORCID 0000-0002-9220-6959

Bruna FS De Sousa ORCID:0000-0003-4125-6611

Jose M Palacios ORCID 0000-0002-2541-8812

Luis Rey ORCID 0000-0003-3477-6942

\section{Abstract}

Many bacteria of the genus Bradyrhizobium are capable of inducing nodules in legumes. In this work, the importance of a type VI secretion system (T6SS) in a symbiotic strain of the genus Bradyrhizobium is described. T6SS of Bradyrhizobium sp. LmicA16 (A16) is necessary for efficient nodulation with Lupinus micranthus and $L$. angustifolius. A mutant in the gene $v g r G$, coding for a component of the T6SS nanostructure, induced less nodules and smaller plants than the wild type strain (wt) and was less competitive when coinoculated with the wt strain. A16 T6SS genes are organized in a $26 \mathrm{~kb}$ DNA region in two divergent gene clusters of nine genes each. One of these genes codes for a protein (Tsb1) of unknown function but containing a methyltransferase domain. A tsbl mutant showed an intermediate symbiotic phenotype regarding $v g r G$ mutant and higher mucoidy and motility than the wt strain in free living conditions. T6SS promoter fusions to the lac $Z$ reporter indicate expression in nodules but not in free living cells grown in different media and conditions. The analysis of nodule structure revealed that the level of nodule colonization was significantly reduced in the mutants with respect to the wt strain.

Keywords Type VI secretion; Rhizobium-legume symbiosis; Bradyrhizobium; Lupinus; effector; Methyltransferase; 


\section{Introduction}

The so-called bacterial type VI secretion system (T6SS) is proving to be of great importance in adaptive activities of bacteria such as competitiveness and interaction with eukaryotes [1,2]. The T6SS is a nanosyringe similar to inverted phage tails generally made up of 13 conserved components called TssA to TssM and able to secrete proteins (effectors) to target cells or to the extracellular milieu [3,4]. It was first described in two human pathogens, Vibrio cholerae and Pseudomonas aeruginosa [5,6] although some of what are now known as tss genes were previously ascribed to protein secretion in a symbiotic Rhizobium leguminosarum strain [7] and in the fish pathogen Edwardsiella tarda [8]. Three main elements form a T6SS: a cytoplasmic baseplate (TssEFGK), a trans-membrane complex formed by TssJLM and a tail. The tail contains an internal tube of hexameric Hcp (also named TssD) rings topped with a spike structure formed by a VgrG (TssI) trimer base and a conical tip PAAR monomer [9] and is wrapped by a contractile sheath made of TssB-C protomers. TssA is required to assemble the Hcp tube and the TssB-C sheath [10]. Components of T6SS are recycled by the ATPase TssH (ClpV) after tail contraction [4]. In addition to Tss core components, T6SS gene clusters encode effectors and accessory proteins refereed as Tag (Type six associated gene) required for assembly of the T6SS subunits and regulation of their expression [4,11]. Most of the effectors described so far are enzymes with antibacterial activity and can degrade the cell wall, DNA or RNA, the cytoplasmic membrane, etc [1]. Often, antibacterial genes are adjacent to cognate immunity ones preventing self-toxicity or toxicity from other bacteria. These genes are known as effector-immunity pairs (E/I pairs) [12] . Other effectors target eukaryots, or act in stress responses or in the acquisition of metals or nutrients from the extracellular milieu and do not require cognate immunity proteins $[2,13]$.

T6SSs are present in $\sim 25 \%$ of Gram-negative bacteria mainly in the Proteobacteria and Bacteroidetes phyla $[3,14]$, among them are plant-associated bacteria including legume symbionts known as rhizobia $[15,16]$. However, there are very few studies about the role of rhizobial T6SSs in symbiosis with legumes and in the rhizosphere. It can be highlighted the aforementioned work by Bladergroen et al in 2003 [7], which showed that the presence of a T6SS impairs the symbiosis with peas of a Rhizobium leguminosarum strain. The main aim of this work was to study the function of the T6SS of a Bradyrhizobium strain that nodulates Lupinus micranthus and L. angustifolius. Lupines belong to the legume tribe Genisteae and are capable of establishing symbioses mainly with Bradyhizobium strains [17]. Bradyrhizobium sp. LmicA16 (A16) was isolated from $L$. micranthus in Northern Algeria. A16 was able to induce effective nodules with its original host and also with L. angustifolius [18]. This led us to obtain a draft of its genome in which a T6SS was identified. In this work it has been shown that the A16 T6SS is required for efficient nodulation with L. micranthus and $L$. angustifolius. It has also been determined that the T6SS is expressed in symbiosis and that one of the potential effector (Tsb1) encoded in the T6SS cluster, presenting a methyltransferase domain, could have a positive role in symbiosis.

\section{Materials and methods}

\section{Strains and culture conditions used in this work}

Strains are listed in Table S1. Escherichia coli was grown in Luria-Bertani (LB) broth at $37^{\circ} \mathrm{C}$ [19]. Rhizobium strains were cultured at $28^{\circ} \mathrm{C}$ in Yeast Mannitol Broth (YMB) [20] or Tryptone Yeast (TY) [21], and selection of transconjugants was carried out in Rhizobium minimal broth (Rmin) [21]. Antibiotic concentrations were ( $\mu \mathrm{g} \mathrm{x} \mathrm{mL} \mathrm{L}^{-1}$ ): $100 \mathrm{Amp}$ (ampicillin), $50 \mathrm{Km}$ (kanamycin), $50 \mathrm{Spc}$ (spectinomycin), $5 \mathrm{Tc}$ (tetracycline), 20 (nalidixic acid), and 20 (cefotaxime).

\section{Construction of vgrG and tsb1 mutants}

The $\operatorname{vg} G$ mutant was done by single recombination with plasmid pK18mobsacB (Table S1) harbouring an internal DNA sequence of $\operatorname{vgr} G$ gene (479 bp). The internal region was amplified by PCR (Table S2), cloned first into pCR2. $1^{\circledR}$-TOPO ${ }^{\circledR}$ and then in $\mathrm{pK} 18 m o b S a c B$ after digestion with EcoRI. E. coli S17.1 cells containing pK18mob- $v g r G$ were used to conjugate the plasmid to A16.

The $t s b 1$ mutant was obtained also by single recombination with plasmid pVO-npt2-cefo-npt2-GFP containing the central region of the gene $t s b 2$ (388 bp) (pVO-tsb1) (Tables S1 and S2). This region was amplified by PCR,

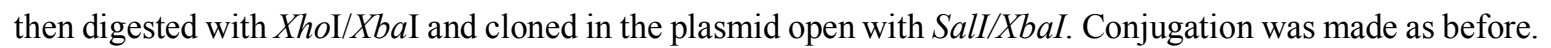


The mutants were selected and purified in Rmin supplemented with antibiotics and checked by PCR.

\section{Complementation of the vgrG mutant}

In order to complement the $\operatorname{vgr} G$ mutant, a DNA fragment of 2,445 bp containing $\operatorname{vgrG}$ and its promoter (Fig. 1) was amplified by PCR (Table S2). The amplicon was cloned in the plasmid pMP220 and conjugated to A16 $\operatorname{vgr} G$-deficient strain using S17.1 (Table S1).

\section{Plant assays and nitrogen fixation activity}

Lupinus micranthus seeds were surface-sterilized in $96 \%$ sulfuric acid for $2 \mathrm{~h}$, washed 10 times with sterile distilled water and kept submerged one day and then placed on water-agar $(1 \%)$ plates at $20^{\circ} \mathrm{C}$ in the dark to germinate. The seeds of $L$. angustifolius were sterilised by immersion in $96^{\circ}$ ethanol for $1 \mathrm{~min}$, in bleach (12\%) for $5 \mathrm{~min}$ and washed 10 times. Seedlings were transferred into sterilized Leonard jars containing vermiculite and Jensen's solution [20] and covered with a $1 \mathrm{~cm}$ thick layer of sterile gravel. Each seedling was inoculated with $1 \mathrm{~mL}$ of a rhizobial suspension $\left(10^{8}-10^{9}\right.$ cells $\left.\mathrm{mL}^{-1}\right)$. Plants were grown at $23-25^{\circ} \mathrm{C}$ in a greenhouse $(16 / 8$ $\mathrm{h}$ day/night) for 5 weeks. At least three different replicates with four plants per replicate were used. Nitrogen fixation activity of nodules estimated by the acetylene reduction test with a Shimadzu GC-8 apparatus have been previously described [22].

\section{A16 T6SS gene expression}

In order to analyse the expression of the T6SS genes of A16, a 690 bp region (P6) comprising $160 \mathrm{bp}$ of the tss $A$ gene, $175 \mathrm{bp}$ of $\operatorname{vgr} G$ and $355 \mathrm{bp}$ of the intergenic region between both genes which likely includes the promoter region for the genes, was amplified with primers shown in Table S2. P6 was fused in front of the promoter lacZ gene of plasmid pMP220 (Table S1). Plasmids containing fusions in the two possible orientations were named P6TssA and P6VgrG, depending on which gene they are oriented towards. P6 derivatives were conjugated to A16 strain. The orientation of the promoter region with respect to the lac $Z$ gene was determined by PCR (Table S2).

P6TssA and P6VgrG expression was studied under different free-living conditions considering different $\mathrm{pHs}$ $(5.5,7,8.5)$, culture medium (Rmin, YMB, TY), temperature $\left(20^{\circ} \mathrm{C}, 28^{\circ}\right)$, presence of root exudates and in bacteroids.

For analysis under free-living conditions, a preinoculum was grown for 5 days at $28^{\circ} \mathrm{C}$. Then, ODs were adjusted to 0.6 and cultures grew for 24 hours. After that, $\beta$-galactosidase activity (OD $540 \times 1000 \times$ t (min) / $\mathrm{OD}_{540} \mathrm{x}$ vol $\left.(\mathrm{mL})\right)$ was measured following the protocol described by Miller (1972) [23].

\section{Preparation of root exudates and growth condition}

Germinated seeds of Lupinus angustifolius, L. micranthus, Phaseolus vulgaris, Leucaena leucocephala, Glycine max, and Triticum aestivum were placed on a $0.8 \mathrm{~mm}$ diameter hollow cylinder so the roots were in contact with Jensen's solution. After four days of submergence, the solution was collected and filtered $(0.2$ $\mu \mathrm{m})$. A16 grew in YMB until $\mathrm{OD}_{600}=0.6$, and then $0.7 \mathrm{~mL}$ of the culture were mixed with $0.3 \mathrm{~mL}$ Jensen's solution containing the different root exudates. Sterilized Jensen's solution was used as negative control. Cultures plus exudates were incubated $3 \mathrm{~h}$ at $28^{\circ} \mathrm{C}$ and then cells were collected by centrifugation for analysis.

\section{Bacteroids extraction}

Extraction of bacteroids was done following the protocol of Ruiz-Argüeso 1978 [22] after five weeks postinoculation with A16 (P6TssA), A16 (P6VgrG) and A16 (pMP220).

\section{Rhizosphere competitive colonization assays}

Tests on the competitive colonisation of the rhizosphere between the wt A16 strain and $\operatorname{vgr} G$ mutant was determined by inoculating Lupinus angustifolius with different ratio $1: 10 ; 1: 1 ; 10: 1$. In order to differentiate the strains, A16 expressed GFP constitutively by harbouring vector pHC60 (Table S1). After 5 weeks, the nodules were counted differently through fluorescence visualisation with NightOWL II LB 983 In Vivo 
Imaging System that allowed differentiation of both strains. In addition, cells from nodules were picked with a sterile loop and streaked on YMB plates to confirm growth with the appropriate antibiotics (tetracyclin for A16 and kanamycin for the $\operatorname{vgr} G$ mutant).

\section{Colony morphology}

In order to compare the appearance of the T6SS mutants the strains were grown in the TY medium up to $\mathrm{OD}_{600}=$ 0.6. Then $10 \mu \mathrm{L}$ of each culture was deposited as a drop in the center of TY and YMB plates. Growth was visualized five days after inoculation. This assay was repeated 3 times with 3 replicates. Viable cells from cultures were counted in Petri dishes and all strains have similar cfu/OD 600 .

\section{Motility assay}

The motility assays is based on the protocol of Liu et al (2012) [24]. A cell suspension (5 $\mu \mathrm{l})$ in TY medium at $\mathrm{OD}_{600}=0.6$ was added to the centre of a TY $0.7 \%$ agar plate, creating an initial droplet of $5 \mathrm{~mm}$ diameter. After 15 days of incubation, the diameter of the colonies was measured. This assay was repeated 2 times independently with 5 replicates.

\section{Bioinformatics analyses and accession numbers}

A16 T6SS was identified from a genomic sequence draft obtained in our group using Illumina HiSeq 2000, 500 bp paired-end libraries, 100 bp reads and 7 million reads. Phylogenetic and molecular evolutionary analyses were conducted using MEGA 7. The NCBI submission number of the A16 T6SS is 2474916.

\section{Confocal laser scanning microscopy and image analysis}

Nodules were washed in PBS and $200 \mu \mathrm{m}$ sections were obtained with a Vibratome 1000 Plus. Microscopy was performed with a laser scanning microscope (Leica SP8). Nodules were observed by monitoring the GFP fluorescence expressed from pHC60 and analyzed with ImageJ.

\section{Results}

\section{T6SS gene cluster organization of Bradyrhizobium sp. LmicA16}

A T6SS was identified in the A16 genome. Twelve of the thirteen tss structural genes (tssA-M), generally conserved in T6SS, were distributed in two divergent clusters. No tss $J$ gene was identified (Fig. 1). The T6SS region also comprises four accessory genes (tagE, $p p p A, \operatorname{tag} F$ and $\operatorname{tag} H$ ) presumably involved in regulation as shown for homologous proteins in other bacteria where the systems are post-translationally regulated, positively by $\operatorname{tag} E$ (Ser-Thr kinase) and negatively by the cognate phosphatase PppA [25]. In A16, PppA and TagF are two distinct proteins while in Rhizobium etli Mim1 they are two domains of the same protein (TagF) (Fig. 1) [26]. In addition to tag accessory genes, two other genes, $t s b 1$ and $t s b 2$, encode proteins of unknown function which could be T6SS-dependent effectors (Fig. 1 and Table 1). It is noteworthy that these genes are not accompanied by other of unknown function which could indicate the presence of a possible E/I pair. Whereas no functional domain has been identified in Tsb2, Tsb1 has a methyltransferase domain (Table 1) identified in silico at Phyre2 web [27]. The analysis identified related structures (100\% confidence) of two protein-lysine methyltransferases (PKMT1 and PKMT2) from Rickettsia typhi [28], with which Tsb1 shares $28 \%$ of sequence identity (coverage $95 \%$ ).

A comparison of T6SS from various rhizobia are presented in Fig. 1 and Table 1. The chosen rhizobia were Bradyhizobium diazoefficiens USDA110, the first sequenced strain of this genus [29] and Azorhizobium caulinodans ORS571 and Rhizobium etli Mim1 for being strains where the role of T6SS in the symbiosis has been studied [26,30]. It was observed that genes tss and tag are present in all strains, but differently organized. T6SS organization and protein similarity of the two Bradyrhizobium are highly conserved. Genes $t s b 1$ and $t s b 2$ of unknown function are not conserved (Table 1).

Bernal et al described in 2018 [16] five T6SS phylogenetic groups based on TssB protein. TssB of A16 belongs to group 3 as TssB from other Bradryrhizobium strains (Fig. 2a). Tsb1 homologous were found mostly associated to T6SS of Bradyrhizobium, Microvirga and Bosea strains and, more distantly from Rickettsia spp. 
(Fig. 2b). Tsb2 is phylogenetically related to T6SS-related proteins from Bradyrhizobium, Bosea and Mesorhizobium (Fig. 2c).

\section{Expression of A16 T6SS}

The DNA region between $v g r G$ and $t s s$ (P6), likely corresponding to the promoter region for the two divergent clusters in T6SS of A16 (Fig. 1) was used to determine the expression of the T6SS A16 in different conditions. P6 was fused in the two possible orientations to the lac $Z$ reporter gene of pMP220 vector generating P6TssA and P6VgrG (Table S1). Their expression in free-living conditions (See Material and Methods) was not detected (data not shown). In bacteroids extracted from L. angustifolius nodules, weak activities (Miller units)

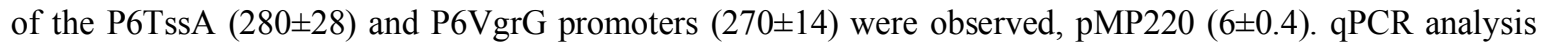
was performed and expression of $h c p, v g r G, t s b 1$ and $t s b 2$ from L. angustifolius bacteroids was negligible compared to rpoD gene (data not shown).

\section{Relevance of A16 T6SS in symbiosis}

In order to evaluate the relevance of the T6SS of A16 in symbiosis, a mutant deficient in $\operatorname{vgr} G$ was generated. VgrG protein is essential for functional T6SS [31]. The growth of $v g r G$ mutant in YMB and TY media showed no difference regarding the wt strain. In order to assess the potential effect of T6SS on the symbiosis, $L$. micranthus and L. angustifolius plants were inoculated with wt and $v g r G$ strains and their symbiotic phenotype were examined. In contrast to the wt strain, no advantage on plant growth could be observed with the $\operatorname{vgr} G$ mutant, i.e. the plants had an aerial dry weight similar to that of the non-inoculated control (Figs. 3 and 4). In addition, the nodules from the mutant were 45-55 \% bigger than those induced by the wt strain, the number and weight of nodules per plant were significantly lower in the $\operatorname{vgr} G$ mutant compared to the wt strain (Figs $3 \mathrm{c}$ and 4d).

Analysis of nitrogen fixation was carried out by the acetylene reduction assay (ARA). This analysis revealed that ARA values were significantly lower in the nodules induced by the vgrG mutant strain in L. angustifolius (Figs. 3 and 4). In Fig. 4c the colonization of the nodules by the different strains is shown and it can be seen that the colonized area is clearly smaller in the nodules induced by $\operatorname{vgr} G$ mutant. This is consistent with the nitrogen fixation values obtained since they are referred to the fresh weight of the nodules and in the mutant nodules, there are clearly fewer bacteroids capable of fixing nitrogen. In order to complement the $v g r G$ mutant, a DNA fragment containing the gene $\operatorname{vgr} G$ and the upstream promoter region designed as P6 were cloned in pMP220 (pvgrG) and conjugated into the $\operatorname{vgr} G$ mutant. The complemented strain approximately reproduced the nodulation and dry weight values of the wt strain (Figs. 3 and 4).

Additionally, the symbiotic effect of the mutant on $t s b 1$ was studied (Figs. 3 and 4). The $t s b 1$ mutant behaved with an intermediate phenotype with respect to the wt and $\operatorname{vgr} G$ mutant, ie, the values regarding the number and weight of nodules and nodule colonization were lower than those of the wt strain but higher than those of the $\operatorname{vgr} G$ mutant. It should be noted that the tsb1 mutant presented a more mucoid appearance than the wt strain and the $\operatorname{vgrG}$ mutant in TY medium (Fig. 5). Studies on T6SS from other bacteria have shown crosstalk between this system and mucoidy and also to motility [32]. A motility analysis was carried out in TY medium with $0.7 \%$ agar and a greater motility could be observed for tsb1 mutant with regarding the wt strain and the $\operatorname{vgrG}$ mutant (Fig. 5).

T6SSs have a wide variety of functions among which is to obtain a fitness advantage against possible bacterial competitors [1.] Therefore, a competitiveness study in symbiosis with L. angustifolius was carried out using wt and $\operatorname{vgr} G$ mutant inoculating at different ratios. Values of nodule occupancy by the two strains showed a lower competitiveness of the $\operatorname{vgr} G$ mutant, indicating that the T6SS would be important for this activity. Thus, when both strains were inoculated in the same proportion, the wt strain occupied $80 \%$ of the nodules and when 10 times more mutant cells were inoculated, the proportion of wt nodules was double that of the nodules occupied by the mutant (Fig. 6).

All these results indicate that T6SS is important for the A16 symbioses.

\section{Discussion}

Rhizobia are key partners for a sustainable agriculture thanks to their ability to fix nitrogen in symbiosis with legumes, which favors a moderate use of nitrogen fertilizers mitigating the negative effects of their excessive utilization [33]. The efficiency of symbiosis varies greatly depending upon various factors such as legume host, 
soil, environment, and rhizobial strains. Several characteristics of rhizobia important in this interaction have been described such as Nod factors, extracellular polysaccharides and secretion systems [34]. There are numerous examples where rhizobial type III or IV secretion systems (T3SS/T4SS) have been found to have neutral, positive or negative effects on symbiosis depending on the species or accession of the host [35]. The role of rhizobial T6SS has been studied only in a few strains although the increasing number of available genomes makes it possible to identify T6SSs in many rhizobia [3,36]. The first report in 2003 indicated that T6SS genes from R. leguminosarum RBL5523 impaired nodulation with peas [7]. In two studies, it was shown that the presence of T6SS had no effect on nodulation but rather did on competitiveness, the cases of Paraburkholderia phymatum/Vigna unguiculata [37] and Azorhizobium caulinodans/Sesbania rostrata [30]. A positive effect of T6SS on symbiosis was recently shown for Rhizobium etli/Phaseolus vulgaris [26].

In this work a positive effect of T6SS on the Bradyrhizobium sp. /Lupinus symbiosis is also demonstrated. The $\operatorname{vgr} G$ mutant strain, lacking the essential structural element $\operatorname{VgrG}$, induced less nodules and the plant aerial dry weight was similar to that of the non-inoculated plants (Figs. 3 and 4). These results are similar to those obtained with other structural mutants in T6SS of $R$. etli, an effective endosymbiont of beans [26]. It is to note that when comparing the T6SS gene clusters of R. etli and that of the A16 (Fig. 1) it can be observed that the structural genes are conserved (although the corresponding percentage of amino acid identity is below $50 \%$, Table 1), but the genes of unknown function, among which the effectors would be found, are different in the two strains. It is unknown how the beneficial effect of these T6SS occurs and whether the mechanism would be similar. The presence of possible T6SS-dependent effectors encoded outside the studied clusters elsewhere in the genomes is also unknown.

So far no T6SS effector targeting legume host cells has been described but it is tempting to speculate that the beneficial role of T6SS in symbiosis is also related to an attenuation of the defense response of the compatible host plant as described by T3SS-dependent rhizobial effectors as effector-triggered susceptibility [38]. As examples, the effector Bel2-5 from Bradyrhizobium elkanii USDA61 is involved in nodule organogenesis and repression of defense genes that impair nodulation in some host genotypes [39], NopC effector from Sinorhizobium fredii HH103 has a positive effect on Glycine max and Vigna unguiculata nodulation [40], NopAB and NopT from Bradyrhizobium vignae ORS3257 are key determinant for nodulation of Vigna spp. [41] and the effector NopM from $S$. fredii NGR234 favours nodulation by reducing plant reactive oxygen species (ROS) during infection [42]. By opposite, negative effects of T3SS effectors in symbiosis can occur if effector-triggered immunity (ETI) of the plant is induced, stopping rhizobial infection $[43,44]$.

Lupinus angustifolius nodule infected cells by the A16 strain and by the $\operatorname{vgr} G$ and $t s b 1$ mutants are shown in Fig. 4c. It can be seen that the infected surface by the wt strain is much larger than that of the $\operatorname{vgr} G$ strain, while the surface area of $t s b 1$ is intermediate. This correlates with the higher nitrogen fixation activity and higher plant size produced with the inoculation of the wt strain compared to $\operatorname{vgr} G$ mutant. The $t s b 1$ mutant showed an in-between phenotype. The fact that the $\operatorname{vgr} G$ mutant has fewer infected cells could be due to the fact that some T6SS-dependent effectors favor infection in a similar way to some T3SS-dependent effectors. In this regard, the effect of the $t s b 1$ mutation suggests that the Tsb1 protein could be a possible T6SS effector having a positive effect on symbiosis. Tsb1 is a protein of unknown function with a methyltransferase domain and whole protein is structurally similar to rickettsial PKMT1 and PKMT2 [28] (Fig. 2b). PKMTs catalyze methylation of the outer membrane protein $\mathrm{OmpB}$ at multiple sites. OmpB is present in all rickettsial species mediating host cell adhesion and invasion, and is also an important antigen. OmpBs from virulent strains have multiple trimethylated lysine residues, whereas the avirulent strains hold mainly monomethyllysine. PKMT1 catalyzes mainly monomethylation and PKMT2 trimethylation and their primary substrate is OmpB, methylating multiple lysyl residues with broad sequence specificity [28]. Recently Engstrom et al (2020) [45] showed that methylation of outer membrane proteins prevents host ubiquitylation and autophagy. This suggests that Tsb1 may methylate some outer membrane proteins favouring interaction with its host legume. However, this is highly speculative because the function of class I methyltransferases which contain a Rossmann fold for binding S-Adenosyl methionine (SAM), and to which PMKT and Tsb1 structurally belong, have very diverse substrate specificities (small molecules, lipids, nucleic acids, etc.) and different target atoms for methylation (nitrogen, oxygen, carbon, sulfur, etc [46]. It should be noted that methyltransferase motifs have been found in other proteins within T6SS clusters of other bacteria although they are structurally diverse and different from Tsb1. Some examples are PF00487 from Rhizobium etli Mim1 [26]; Azc_2606 and Azc_2704; from A. caulinodans [30] and Tfe7 homologous to the ribosomal RNA large subunit methyltransferase D required for the fully methylation of $23 \mathrm{~S}$ ribosomal RNA [47]. Interestingly, no gene encoding an immunity pair was found linked to these genes, suggesting a distinct activity of the antibacterial effectors as is the case of antifungal 
effectors described in the opportunistic pathogen Serratia marcescens [48]. On the other hand, the tsb1 mutant has a more mucoid morphological appearance than the parental strain or the $\operatorname{vgr} G$ mutant and also higher motility. Although relationships between T6SS, motility and biofilm production have been described [32] the relationship of Tsb1 to these processes requires further investigation.

The T6SS region in A16 is probably post-translationally regulated by the Ser-Thr kinase $\operatorname{tag} E$ (positively) and negatively by the cognate phosphatase PppA [25]. In A16 PppA and TagF are two distinct proteins while in Agrobacterium tumefaciens and in Rhizobium etli they are two domains of the same protein (TagF). TagF and TagH are encoded in A16 T6SS cluster, the interaction of these two proteins has been demonstrated critical for T6SS repression in A. tumefaciens and Pseudomonas aeruginosa [25,49]. Bacteria deploy TagE-dependent activation and TagF-mediated repression mechanisms to control T6SS but the environmental signals to activate the system are not identified. In this work we have found low expression in nodules and no free-living activity under the conditions tested. This suggests an early role in symbiosis. The role of T6SS in free-living conditions is still unknown but it would be involved in interbacterial competition, as has been demonstrated in A16 strain and in those already mentioned of $A$. caulinodans [30] and P. phymatum [37]. The capacity of T6SS from phytobacteria to outcompete other bacteria has also been seen in Pseudomonas spp., Pantoea ananatis, A. tumefaciens etc $[15,16]$.

To our knowledge, this is the first time that a T6SS of a symbiont of the genus Bradyrhizobium has been shown to be necessary for effective symbiosis with legumes. Although possible functions have been suggested for tsb1 gene, future work is needed to identify and characterize T6SS-dependent effectors and to understand the specific role they can play in symbiosis and/or in the rhizosphere. This information can be of great use in fields such as ecology, agriculture, and medicine.

\section{Supplementary information}

Tables S1 and S2

\section{Author Contributions:}

LR, FB and LT designed the study. The experiments are performed by LT. LT, BFSS and LR analysed the data. LT an LR wrote the initial draft and all authors contributed to manuscript revision and approved it to be published.

Acknowledgements: We thank Carlos González Carneros for their assistance with Microscopy work and Rodrigo Gómez Pellicer for technical support.

Funding: This research was funded by the Ministerio de Ciencia, Innovación y Universidades, Spain (RTI2018-094985-B-100). BFSS is supported by a scholarship from CNPq-GDE-204842/2018-2. L.T. is supported by scholarships from the National Exceptional Program (PNE), Ministry of Higher Education and Scientific research of Algeria and from the Erasmus+ Programme project 2019-1-ES01-KA107-063778. EG is supported by the ANR grants 'SymEffectors' and 'ET-Nod' (ANR- 16-CE20-0013 and ANR-20-CE20-0012, respectively).

Conflict of interest: The authors declare no conflict of interest. The funders had no role in the design of the study, the collection, analyses, or interpretation of data, writing of the manuscript, or the decision to publish the results

\section{References}

1. Hernandez RE, Gallegos-Monterrosa R, Coulthurst SJ (2020) Type VI secretion system effector proteins: Effective weapons for bacterial competitiveness. Cell Microbiol. 2(9). doi:10.1111/cmi.13241

2. Monjarás Feria J, Valvano MA. An Overview of Anti-Eukaryotic T6SS Effectors (2020) Front Cell Infect Microbiol. 10. doi:10.3389/fcimb.2020.584751

3. Boyer F, Fichant G, Berthod J, Vandenbrouck Y, Attree I (2009) Dissecting the bacterial type VI secretion system by a genome wide in silico analysis: what can be learned from available microbial genomic resources? BMC Genomics. 10:104. doi:10.1186/1471-2164-10-104

4. Silverman JM, Brunet YR, Cascales E, Mougous JD (2012) Structure and regulation of the type VI secretion system. Annu Rev Microbiol. 66:453-472. doi:10.1146/annurev-micro-121809-151619

5. Mougous JD et al (2006) A Virulence Locus of Pseudomonas aeruginosa Encodes a Protein Secretion 
6. Pukatzki S, Ma AT, Sturtevant D, et al (2006) Identification of a conserved bacterial protein secretion system in Vibrio cholerae using the Dictyostelium host model system. Proc Natl Acad Sci. 103:15281533. doi:10.1073/pnas.0510322103

7. Bladergroen MR, Badelt K, Spaink HP (2003) Infection-Blocking Genes of a Symbiotic Rhizobium leguminosarum Strain That Are Involved in Temperature-Dependent Protein Secretion. Mol PlantMicrobe Interact. 16:53-64. doi:10.1094/MPMI.2003.16.1.53

8. Srinivasa Rao PS, Yamada Y, Tan YP, Leung KY (2004) Use of proteomics to identify novel virulence determinants that are required for Edwardsiella tarda pathogenesis. Mol Microbiol. 53:573-586. doi:10.1111/j.1365-2958.2004.04123.x

9. Wang J, Brodmann M, Basler M (2019) Assembly and Subcellular Localization of Bacterial Type VI Secretion Systems. Annu Rev Microbiol. 73:621-638. doi:10.1146/annurev-micro-020518-115420

10. Dix SR, Owen HJ, Sun R, et al (2018) Structural insights into the function of type VI secretion system TssA subunits. Nat Commun. 9:4765. doi:10.1038/s41467-018-07247-1

11. Shalom G, Shaw JG, Thomas MS (2007) In vivo expression technology identifies a type VI secretion system locus in Burkholderia pseudomallei that is induced upon invasion of macrophages. Microbiology. 153:2689-2699. doi:10.1099/mic.0.2007/006585-0

12. Yang X, Long M, Shen X (2018) Effector-Immunity Pairs Provide the T6SS Nanomachine its Offensive and Defensive Capabilities. Molecules. 23:1009. doi:10.3390/molecules23051009

13. Yu K-W, Xue P, Fu Y, Yang L (2021) T6SS Mediated Stress Responses for Bacterial Environmental Survival and Host Adaptation. Int J Mol Sci. 22:478. doi:10.3390/ijms22020478

14. Böck D, Medeiros JM, Tsao H-F, et al (2017) In situ architecture, function, and evolution of a contractile injection system. Science (80- ). 357:713-717. doi:10.1126/science.aan7904

15. Ryu C-M (2015) Against friend and foe: Type 6 effectors in plant-associated bacteria. J Microbiol. 53:201-208. doi:10.1007/s12275-015-5055-y

16. Bernal P, Llamas MA, Filloux A (2018) Type VI secretion systems in plant-associated bacteria. Environ Microbiol. 20:1-15. doi:10.1111/1462-2920.13956

17. Stępkowski T, Banasiewicz J, Granada C, Andrews M, Passaglia L (2018) Phylogeny and Phylogeography of Rhizobial Symbionts Nodulating Legumes of the Tribe Genisteae. Genes (Basel). 9:163. doi:10.3390/genes9030163

18. Bourebaba Y, Durán D, Boulila F, et al (2016) Diversity of Bradyrhizobium strains nodulating Lupinus micranthus on both sides of the Western Mediterranean: Algeria and Spain. Syst Appl Microbiol. 39:266274. doi:10.1016/j.syapm.2016.04.006

19. Sambrook J, Fritsc E, Maniatis T (1989) Molecular Cloning: A Laboratory Manual. New York, NY: Cold Spring Harbor Laboratory Press MacKenzie SL, Lapp MS, Child JJ (1979) Fatty acid composition of Rhizobium spp. Can J Microbiol. 25:68-74. doi:10.1139/m79-011

20. J. M. Vincent (1972) J. M. Vincent, A Manual for the Practical Study of the Root-Nodule Bacteria (IBP Handbuch No. 15 des International Biology Program, London). XI u. 164 S., 10 Abb., 17 Tab., 7 Taf. Oxford-Edinburgh 1970: Blackwell Scientific Publ., 45 s. Z Allg Mikrobiol. 12:440-440. doi:10.1002/jobm.19720120524

21. O'Gara F, Shanmugan KT (1976) Regulation of nitrogen fixation by rhizobia: export of fixed nitrogen as NH4. Biochim Biophys Acta 437:313-21

22. Ruiz-Argueso T, Hanus J, Evans HJ (1978) Hydrogen production and uptake by pea nodules as affected by strains of Rhizobium leguminosarum. Arch Microbiol. 116:113-118. doi:10.1007/BF00406025

23. Miller, J. H., Mills, A. D., Milton, D. L., Mizuno, S., Montarras, D., Miiller, C. W., et al (1972) Miller JH. Experiments in molecular genetics. Cold Spring Harbor Laboratory Press, Cold Spring Harbor, NY

24. Liu H, Tian W-X, Ibrahim M, et al (2012) Characterization of pilP, a gene required for twitching motility, pathogenicity, and biofilm formation of Acidovorax avenae subsp. avenae RS-1. Eur J Plant Pathol. 134:551-560. doi:10.1007/s10658-012-0038-x

25. Lin J-S, Pissaridou P, Wu H-H, Tsai M-D, Filloux A, Lai E-M (2018) TagF-mediated repression of bacterial type VI secretion systems involves a direct interaction with the cytoplasmic protein Fha. J Biol Chem. 293:8829-8842. doi:10.1074/jbc.RA117.001618

26. Salinero-Lanzarote A, Pacheco-Moreno A, Domingo-Serrano L, et al (2019) The Type VI secretion system of Rhizobium etli Mim1 has a positive effect in symbiosis. FEMS Microbiol Ecol. 95. doi:10.1093/femsec/fiz054

Kelley LA, Mezulis S, Yates CM, Wass MN, Sternberg MJE (2015) The Phyre2 web portal for protein 
modeling, prediction and analysis. Nat Protoc. 10:845-858. doi:10.1038/nprot.2015.053

28. Abeykoon AH, Noinaj N, Choi B-E, et al (2016) Structural Insights into Substrate Recognition and Catalysis in Outer Membrane Protein B (OmpB) by Protein-lysine Methyltransferases from Rickettsia. $J$ Biol Chem. 291:19962-19974. doi:10.1074/jbc.M116.723460

29. Kaneko T(2002) Complete Genomic Sequence of Nitrogen-fixing Symbiotic Bacterium Bradyrhizobium japonicum USDA110. DNA Res. 9:189-197. doi:10.1093/dnares/9.6.189

30. Lin H-H, Huang H-M, Yu M, Lai E-M, Chien H-L, Liu C-T (2018) Functional Exploration of the Bacterial Type VI Secretion System in Mutualism: Azorhizobium caulinodans ORS571- Sesbania rostrata as a Research Model. Mol Plant-Microbe Interact. 31:856-867. doi:10.1094/MPMI-01-18-0026R

31. Lin J-S, Ma L-S, Lai E-M (2013) Systematic Dissection of the Agrobacterium Type VI Secretion System Reveals Machinery and Secreted Components for Subcomplex Formation. Roujeinikova A, ed. PLoS One. 8:e67647. doi:10.1371/journal.pone.0067647

32. Bouteiller M, Gallique M, Bourigault Y, et al (2020) Crosstalk between the Type VI Secretion System and the Expression of Class IV Flagellar Genes in the Pseudomonas fluorescens MFE01 Strain. Microorganisms. 8:622. doi:10.3390/microorganisms8050622

33. Santos MS, Nogueira MA, Hungria M (2019) Microbial inoculants: reviewing the past, discussing the present and previewing an outstanding future for the use of beneficial bacteria in agriculture. $A M B$ Express. 9:205. doi:10.1186/s13568-019-0932-0

34. Walker L, Lagunas B, Gifford ML (2020) Determinants of Host Range Specificity in Legume-Rhizobia Symbiosis. Front Microbiol. 11. doi:10.3389/fmicb.2020.585749

35. Nelson MS, Sadowsky MJ. Secretion systems and signal exchange between nitrogen-fixing rhizobia and legumes. Front Plant Sci. 2015;6. doi:10.3389/fpls.2015.00491

36. Sugawara M, Epstein B, Badgley BD, et al (2013) Comparative genomics of the core and accessory genomes of 48 Sinorhizobium strains comprising five genospecies. Genome Biol. 14:R17. doi:10.1186/gb-2013-14-2-r17

37. De Campos SB, Lardi M, Gandolfi A, Eberl L, Pessi G (2017) Mutations in Two Paraburkholderia phymatum Type VI Secretion Systems Cause Reduced Fitness in Interbacterial Competition. Front Microbiol. 8. doi:10.3389/fmicb.2017.02473

38. Berrabah F, Ratet P, Gourion B (2019) Legume Nodules: Massive Infection in the Absence of Defense Induction. Mol Plant-Microbe Interact. 32:35-44. doi:10.1094/MPMI-07-18-0205-FI

39. Ratu STN, Teulet A, Miwa H, et al (2021) Rhizobia use a pathogenic-like effector to hijack leguminous nodulation signalling. Sci Rep. 11:2034. doi:10.1038/s41598-021-81598-6

40. Jiménez-Guerrero I, Pérez-Montaño F, Medina C, Ollero FJ, López-Baena FJ (2015) NopC Is a Rhizobium-Specific Type 3 Secretion System Effector Secreted by Sinorhizobium (Ensifer) fredii HH103. Mergaert P, ed. PLoS One. 10:e0142866. doi:10.1371/journal.pone.0142866

41. Songwattana P, Chaintreuil C, Wongdee J, et al (2021) Identification of type III effectors modulating the symbiotic properties of Bradyrhizobium vignae strain ORS3257 with various Vigna species. Sci Rep. 11:4874. doi:10.1038/s41598-021-84205-w

42. Xin D-W, Liao S, Xie Z-P, et al (2012) Functional Analysis of NopM, a Novel E3 Ubiquitin Ligase (NEL) Domain Effector of Rhizobium sp. Strain NGR234. Chang J, ed. PLoS Pathog. 8:e1002707. doi:10.1371/journal.ppat.1002707

43. Yasuda M, Miwa H, Masuda S, Takebayashi Y, Sakakibara H, Okazaki S (2016) Effector-Triggered Immunity Determines Host Genotype-Specific Incompatibility in Legume- Rhizobium Symbiosis. Plant Cell Physiol. 57:1791-1800. doi:10.1093/pcp/pcw104

44. Jiménez-Guerrero I, Acosta-Jurado S, Medina C, et al (2020) The Sinorhizobium fredii HH103 type III secretion system effector NopC blocks nodulation with Lotus japonicus Gifu. Gifford M, ed. J Exp Bot. 7:6043-6056. doi:10.1093/jxb/eraa297

45. Engström P, Burke TP, Tran CJ, Iavarone AT, Welch MD (2021) Lysine methylation shields an intracellular pathogen from ubiquitylation and autophagy. Sci Adv. 7:eabg2517. doi:10.1126/sciadv.abg2517

46. Sun Q, Huang M, Wei Y (2021) Diversity of the reaction mechanisms of SAM-dependent enzymes. Acta Pharm Sin B. 11:632-650. doi:10.1016/j.apsb.2020.08.011

47. Durán D, Bernal P, Vazquez-Arias D, et al (2021) Pseudomonas fluorescens F113 type VI secretion systems mediate bacterial killing and adaption to the rhizosphere microbiome. Sci Rep. 11:5772. doi:10.1038/s41598-021-85218-1 
48. Trunk K, Peltier J, Liu Y-C, et al (2018) The type VI secretion system deploys antifungal effectors against microbial competitors. Nat Microbiol. 3:920-931. doi:10.1038/s41564-018-0191-x

49. Chen L, Zou Y, She P, Wu Y (2015) Composition, function, and regulation of T6SS in Pseudomonas aeruginosa. Microbiol Res. 172:19-25. doi:10.1016/j.micres.2015.01.004 
Table 1. Core and accessory gene754sof A16 T6SS and putative functions. Comparison to $R$.

495 etliMim1,B.diazoefficiens USDA110 and to A. caulinodansORS_571

\begin{tabular}{|c|c|c|c|c|c|c|c|c|c|c|c|}
\hline \multicolumn{2}{|c|}{$\begin{array}{l}\text { Bradyrhizobium } \\
\text { sp. LmicA16 }\end{array}$} & \multirow[t]{2}{*}{ Strains } & \multicolumn{3}{|c|}{$\begin{array}{l}\text { B. diazoefficiens } \\
\text { USDA110 }\end{array}$} & \multicolumn{3}{|c|}{$\begin{array}{l}\text { Rhizobium } \\
\text { etli Mim1 }\end{array}$} & \multicolumn{3}{|c|}{$\begin{array}{c}\text { Azorhizobium } \\
\text { caulinodans ORS571 }\end{array}$} \\
\hline \multicolumn{2}{|c|}{ T6SS genes $(\mathbf{a a})^{1}$} & & \multicolumn{3}{|c|}{$\operatorname{ORF}(\mathbf{a a})(\%)^{2}$} & \multicolumn{3}{|c|}{ ORF $(\mathrm{aa})(\%)^{2}$} & \multicolumn{3}{|c|}{ ORF (aa) $(\%)^{2}$} \\
\hline$\overline{\operatorname{tag} E}$ & $(602)$ & Ser/Thr protein kinase & $\operatorname{tag} E$ & $(602)$ & $(86)$ & $\operatorname{tag} E$ & $(303)$ & (31) & & & \\
\hline pppA & (258) & $\begin{array}{l}\text { Ser/Thr phosphatase } \\
\text { type } 2\end{array}$ & blr3603 & (259) & $(85)$ & $\operatorname{tagF}$ & $(455 \mathrm{Ct})$ & (24) & & & \\
\hline $\operatorname{tag} F$ & (233) & DUF2094 & $\operatorname{tag} F$ & (234) & (79) & $\operatorname{tag} F$ & $(455 \mathrm{Nt})$ & (29) & & & \\
\hline$t s s M$ & (1182) & Integral membrane & $t s s M$ & (1179) & $(92)$ & $t s s M$ & (1158) & (31) & $t s s M$ & $(1178)$ & $(28)$ \\
\hline$t s s L$ & (508) & Integral membrane & $t s s L$ & (509) & (89) & $t s s L$ & $(512)$ & (34) & tssL & $(464)$ & $(44)$ \\
\hline$t s s K$ & $(445)$ & Baseplate complex & $t s s K$ & $(450)$ & (94) & $t s s K$ & $(446)$ & (35) & $t s s K$ & $(444)$ & $(38)$ \\
\hline $\operatorname{tag} H$ & (458) & FHA domain & $\operatorname{tag} H$ & (461) & (77) & $\operatorname{tag} H$ & (394) & (32) & & & \\
\hline$t s b 2^{3}$ & (188) & Unknown & blr3597 & (188) & (87) & - & & & - & & \\
\hline$t s s I / v g r G$ & $(622)$ & Puncturing device & $t s s I$ & $(623)$ & (95) & tssI & $(754)$ & (34) & $t s s I$ & $(680)$ & (35) \\
\hline tss $A$ & (437) & Baseplate complex & tss $A$ & (447) & $(81)$ & $t s s A$ & $(354)$ & (36) & $t s s A$ & $(381)$ & (51) \\
\hline$t s s B$ & (175) & Cytoplasmic sheath & $t s s B$ & (184) & (94) & $t s s B$ & $(181)$ & (47) & $t s s B$ & $(170)$ & (41) \\
\hline tss $C$ & (500) & Cytoplasmic sheath & $t s s C$ & (503) & (98) & $t s s C$ & (493) & (48) & $t s s C$ & (494) & (48) \\
\hline$t s s D / h c p$ & (161) & Secretiontube & $t s s D$ & (162) & (94) & $t s s D$ & (158) & (23) & $T s s D$ & $(158)$ & (29) \\
\hline tss $E$ & (182) & Baseplate complex & $t s s E$ & (183) & (84) & $t s s E$ & (169) & (21) & $t s s E$ & $(150)$ & (30) \\
\hline$t s s F$ & $(655)$ & Baseplate complex & $t s s F$ & (444) & (89) & $t s s F$ & $(593)$ & (30) & $t s s F$ & $(607)$ & (30) \\
\hline$t s s G$ & (354) & Baseplate complex & $t s s G$ & (355) & $(88)$ & $\operatorname{tss} G$ & (333) & (26) & $t s s G$ & $(356)$ & (29) \\
\hline$t s s H(c l p V)$ & (878) & Disassembler ATPase & $t s s H$ & $(880)$ & (91) & $t s s H$ & (919) & (49) & $t s s H$ & $(875)$ & (50) \\
\hline$t s b 1^{3}$ & (528) & SAM-Methyltransferase & bll3586 & (533) & (83) & PF00487 & (84) & - & Azc-2606 & (255) & (24) \\
\hline
\end{tabular}

$497{ }^{1}$ Gene names tss (type six secretion) and tag (T6SS-associated gene) were proposed by Shalom et al.

$498 \quad 2007 .^{2}$ Amino acid identity to A16 strain.

$499 \quad{ }^{3}$ Names used in this work after Type six secretion $\underline{B}$ radyrhizobium (tsb) 
504

505

506

507

508

509

510

511

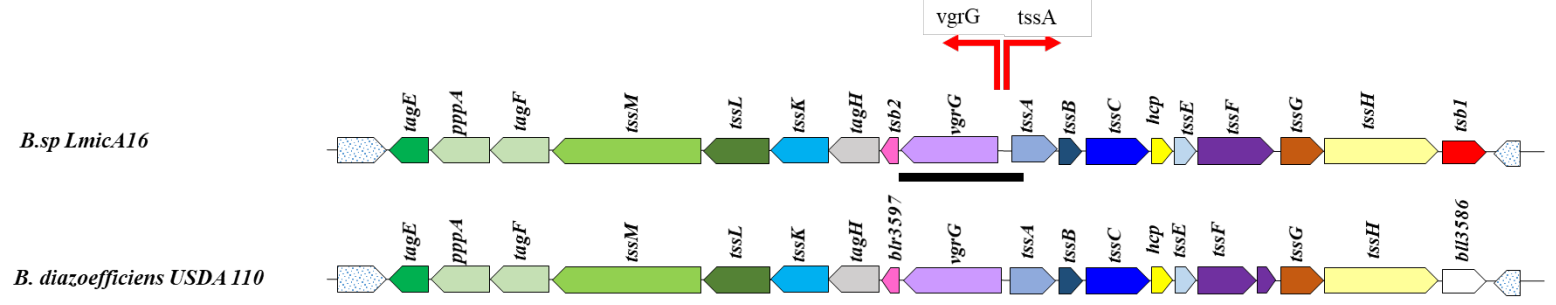

512

513

Fig. 1 Genetic organization of Bradyrhizobium sp. LmicA16 T6SS cluster and homologous region of $B$. diazoefficiens USDA110, A. caulinodans ORS571 and $R$. etli Mim1. Two divergent clusters named vgrG and tss A are indicated by red arrows. Orthologous genes show the same color; white genes correspond to non-conserved genes. The black bar under $\operatorname{vgr} G$ shows the amplified region to complement vgrG mutant.

519

520

521

522

523

524

525

526

527 
a

538

539

540

541

542

543

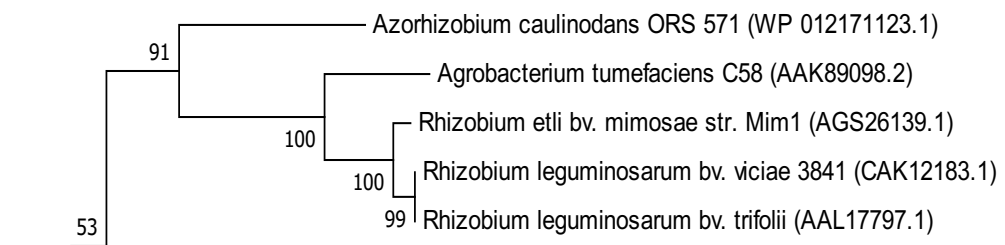

- Agrobacterium tumefaciens C58 (AAK89098.2)

Rhizobium leguminosarum bv. viciae 3841 (CAK12183.1)

Pseudomonas putida S16 (AEJ13387.1)

83 Pseudomonas taiwanensis SJ9 (WP 024087179.1)

Pseudomonas sp. BG2dil (MBG6128331.1)

L Pseudomonas syringae pv. syringae B728a (WP 003318241.1)

93

Burkholderia vietnamiensis G4 (WP 212164368.1)

Rhizobium etli CIAT 652 (WP 040111818.1)

Pantoea ananatis LMG 20103 (ADD77533.1)

545

546

547

548

549

550

551

552

553

554

Mesorhizobium loti MAFF303099 (WP 010910843.1)

54 - Bradyrhizobium japonicum USDA 6 (AHY53440.1)

97 Pseudomonas fluorescens Pf29Arp (WP 005618110.1) Pseudomonas putida W619 3261 (WP 119687440.1) Pantoea ananatis LMG 20103 (ADD79318.1)

$100 \begin{array}{r}\text { Burkholderia thailandensis E264 (WP 009896198.1) } \\ 100 \Gamma^{\text {Pectobacterium wasabiae WPP163 (WP 005972057.1) }}\end{array}$

100

Pectobacterium carotovorum subsp. carotovorum PC1 (WP 015841410.1)

- Pseudomonas fluorescens Pf29Arp (WP 030139161.1)

Pseudomonas syringae DC3000 (WP 011104071.1)

100 Pseudomonas syringae pv. tomato T1 (WP 011104071.1)

555

0.10

556

557

558

559

560

561

562 


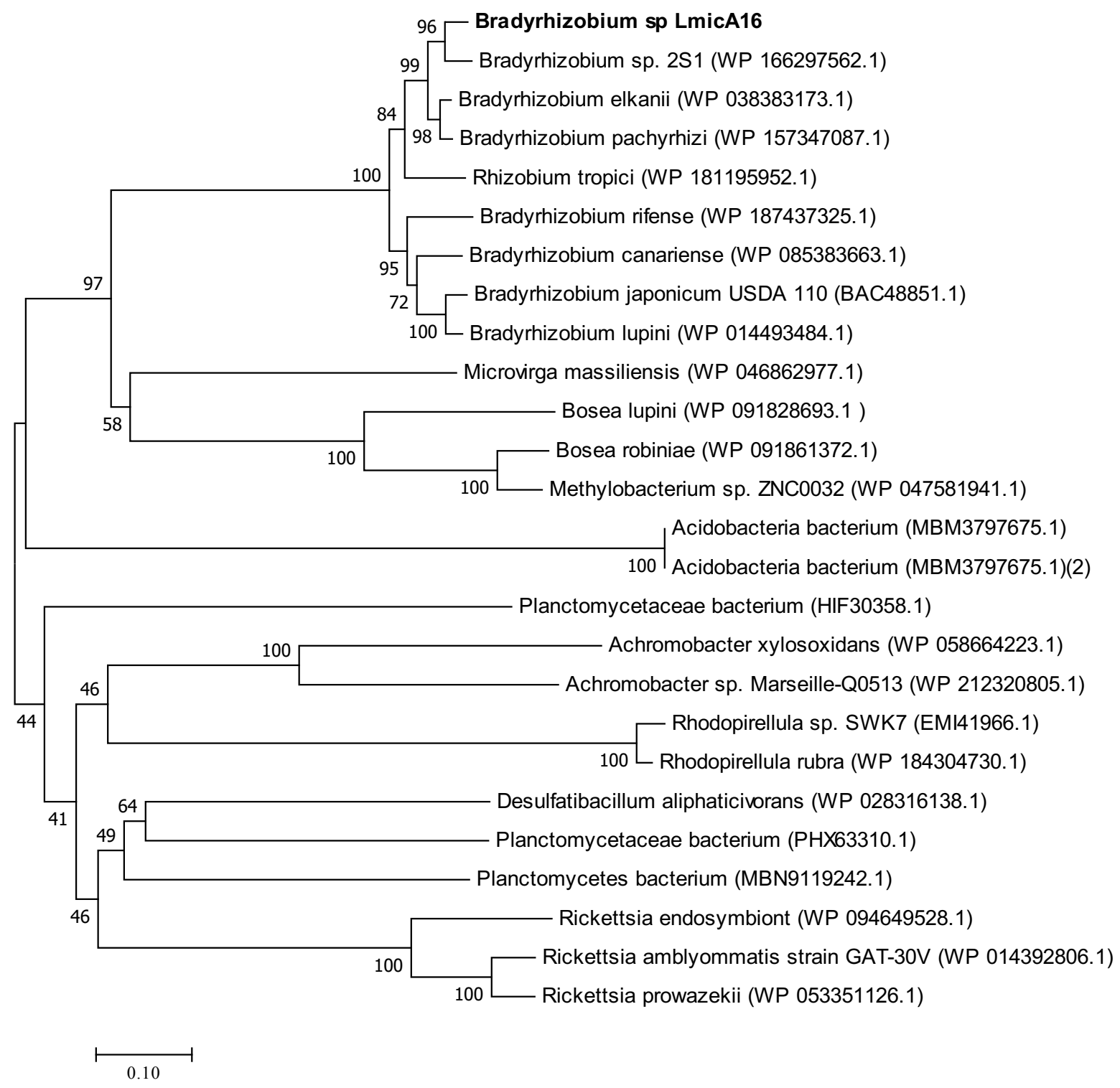


579

581

582

583

584

585

586

587

588

589

590

591

592

593

594

595

596

597

598

599

600

601

602

603

604

605

606

607

\section{c}
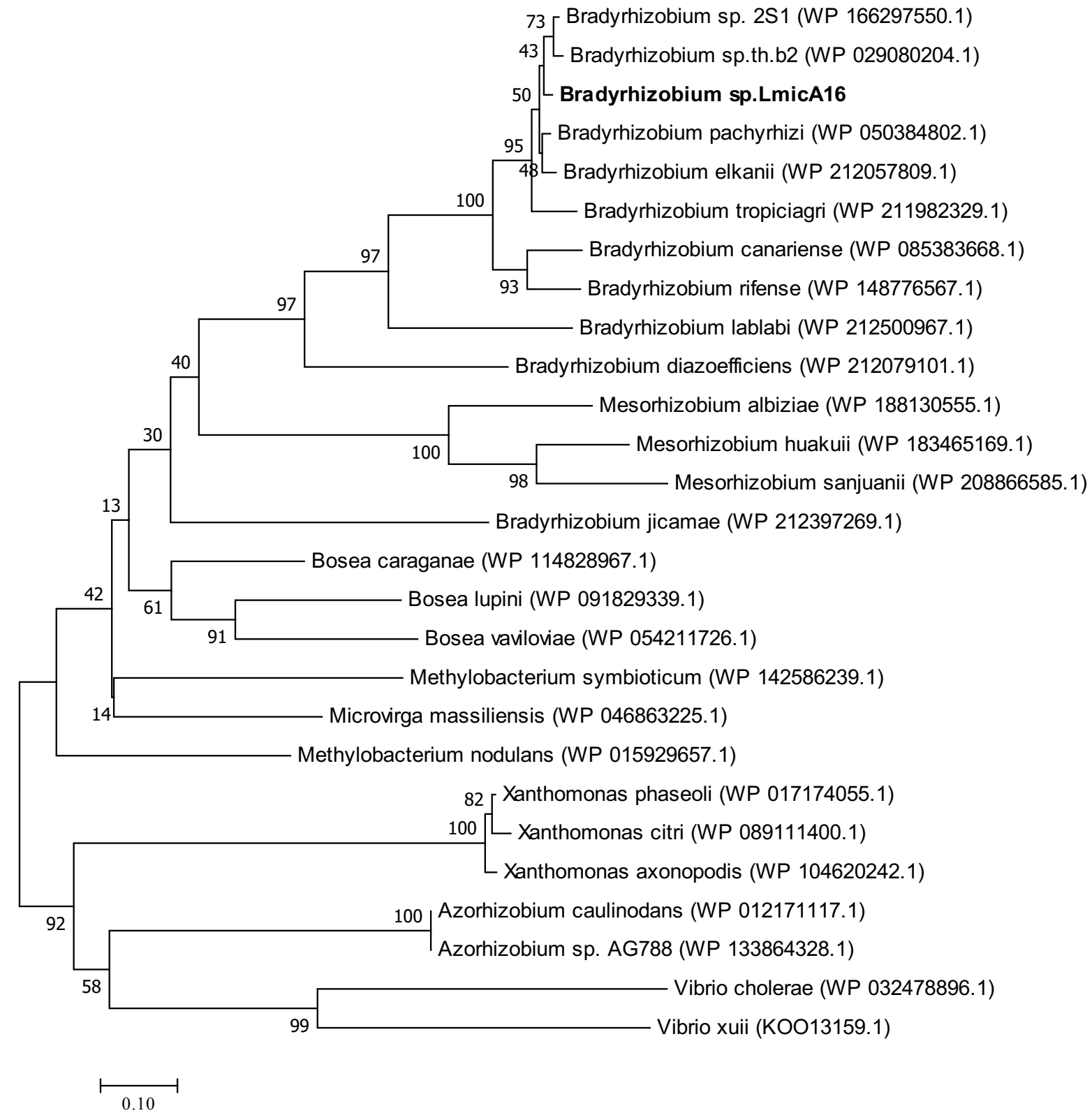

Fig. 2 Neighbor-joining phylogenetic trees based on proteins TssB, Tsb1 and Tsb2. (a), Neighborjoining phylogenetic tree based on the TssB protein. The five groups were determined according to the phylogenetic distribution of T6SS clusters in plant-associated bacteria by Bernal et al. (2018) [17]. (b) phylogenetic tree based on Tsb1 (c) Phylogenetic tree based on Tsb2. Bootstrap values using 1000 replicates are indicated at branching points. The accession numbers are shown in brackets. Bars: 0.1 estimated substitutions. 
a

609

610

611

612

613

614

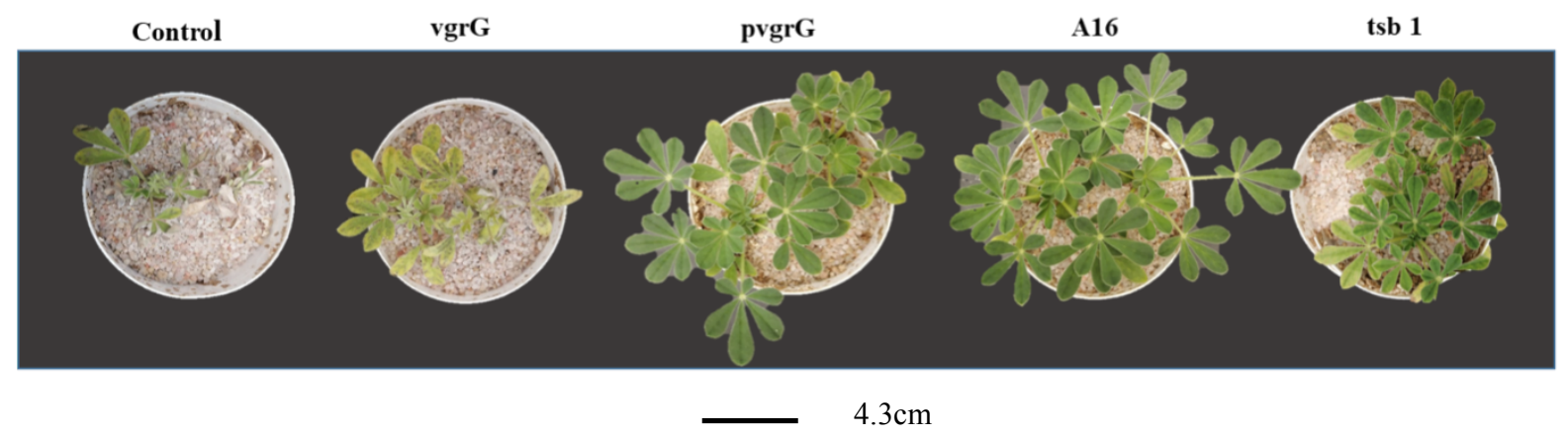

615 b

616

617

618

619

620

621

622
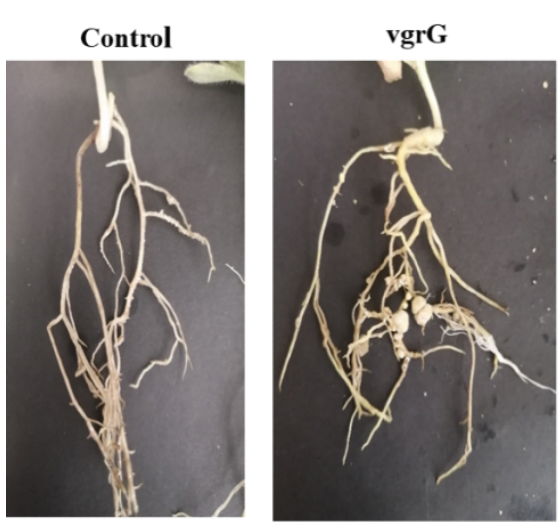

pvgrG

A16

tsb 1
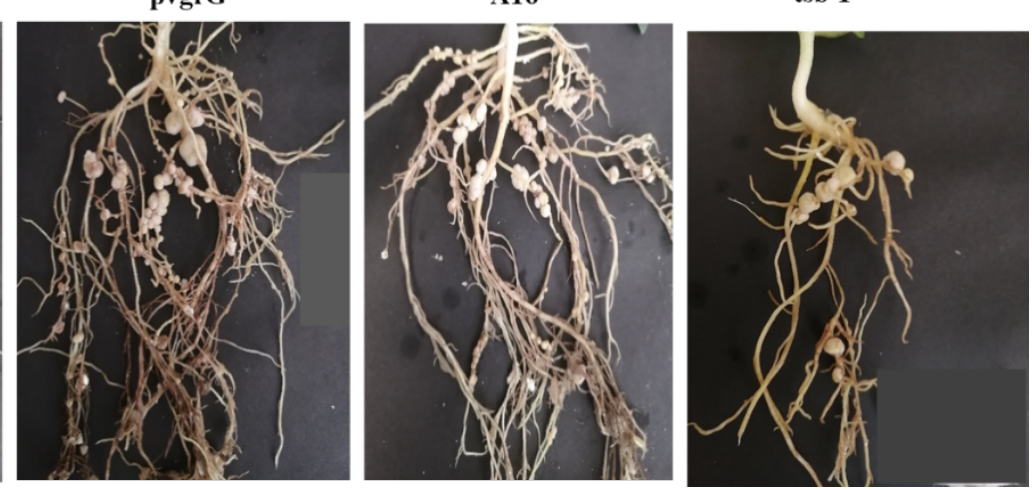

623

624

c

625

626

627

628
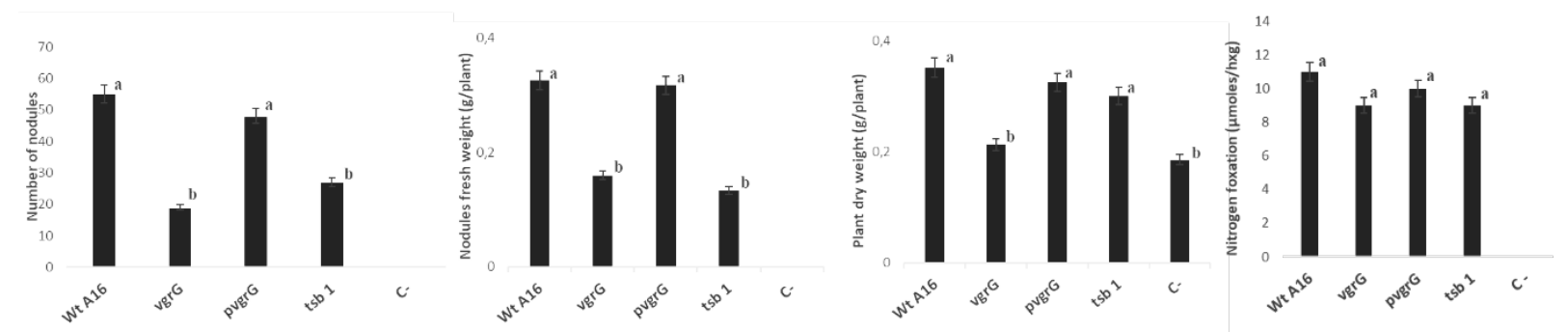

629

630

Fig. 3 Symbiotic phenotype of A16 and derivatives with L. micranthus 5 weeks after inoculation. C-

632 (control) correspond to uninoculated plant. (a) Aerial part. (b) Roots and nodules. (c) Quantitative data

633 from nodules and dry weight of plants. Values are the average of at least 12 plants from 3 replicates (4

634 plants/replicate). Values with the same letter are not significantly different using a Student's test

635 $(\mathrm{p}=0.05)$. 


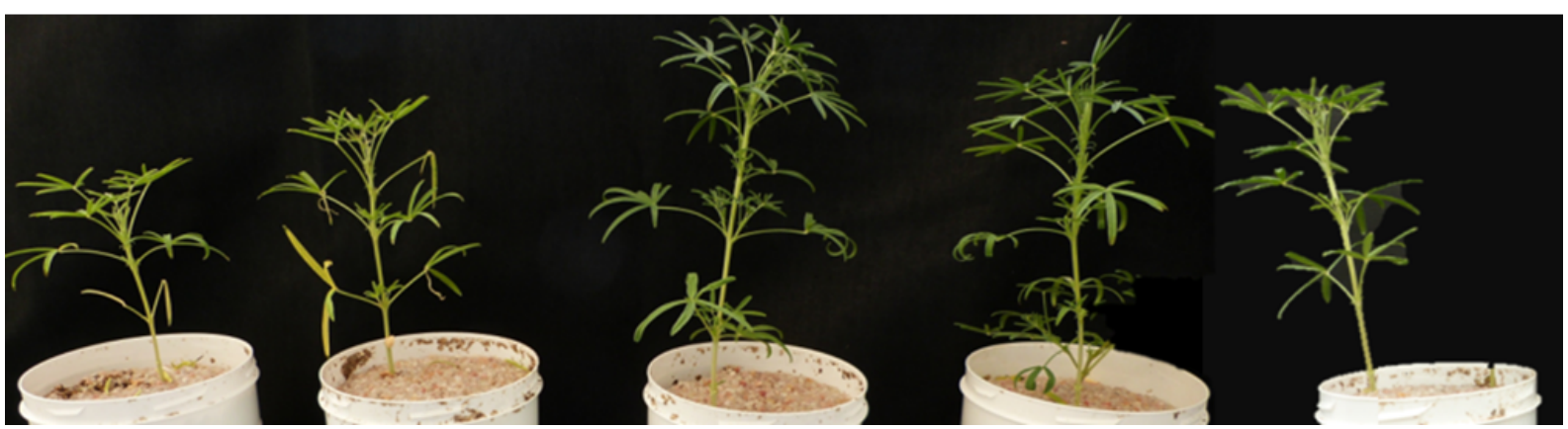

vgrG

pvgrG

$4.3 \mathrm{~cm}$

644
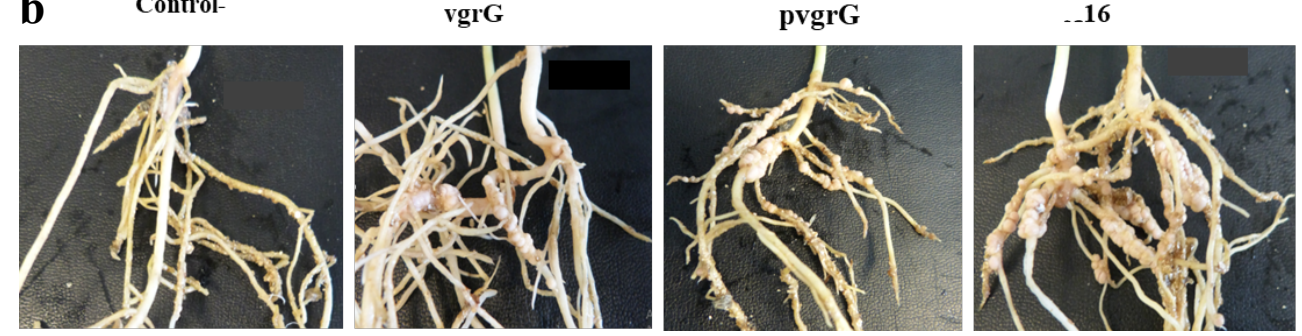

tsb 1

645

646

647

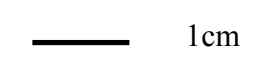

c

Wt A16

vgrG

tsb 1

649

650

651

652

653

654
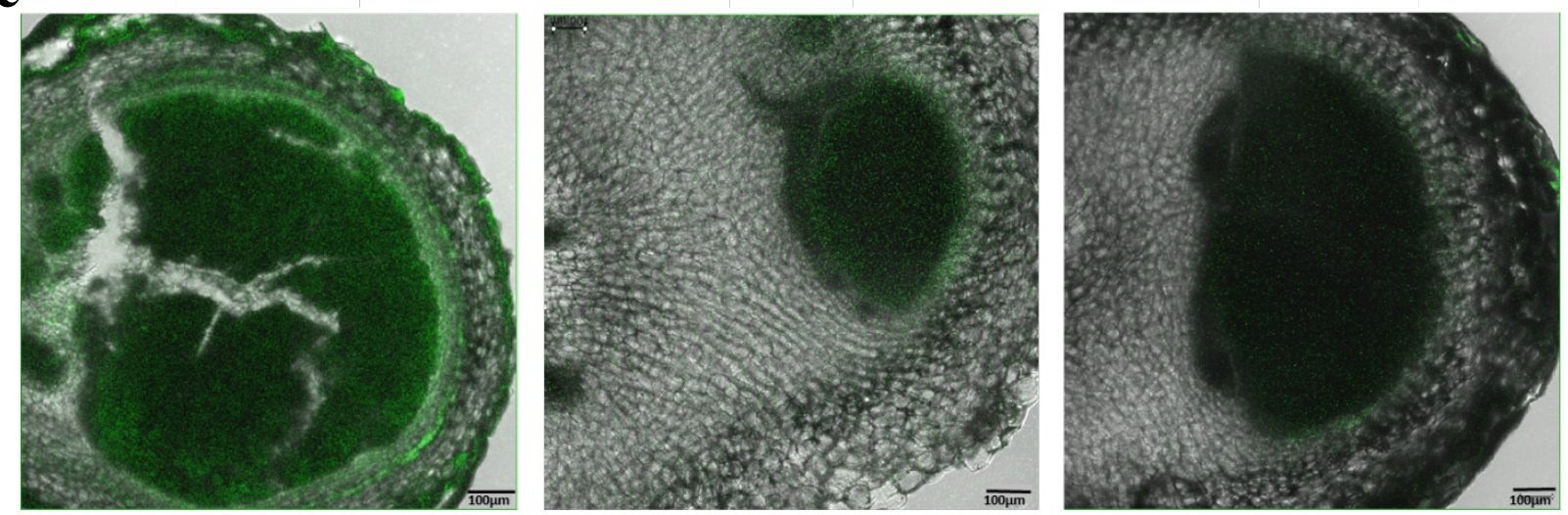

655

d

657

658

659

660

661

Fig. 4 Symbiotic phenotype of A16 and derivatives with L. angustifolius 5 weeks after inoculation. C(control) correspond to uninoculated plant. (a) Aerial part. (b) Roots and nodules. (c) Nodule structure induced by A16, $\operatorname{vgr} G$ and $t s b 1$ strains harboring plasmid pHC60 expressing GFP protein constitutively. (d) Quantitative data from nodules and dry weight of plants. Values are the average of at least 12 plants from 3 replicates (4 plants/replicate). Values with the same letter are not significantly different using a Student's test $(\mathrm{p}=0.05)$. 
669 a

670

671

672

673

674

675

676

677

678 Fig. 5 Colony morphology and motility of A16 mutants vgrG and tsb1 mutants in different media

679 (YMB, TY). (a) $10 \mu \mathrm{l}$ culture of $1,6.10^{8} \pm 0.1 \mathrm{CFU} \cdot \mathrm{mL}-1$ was spotted on the plates. (b) $5 \mu 1$ of the same 680

681 b

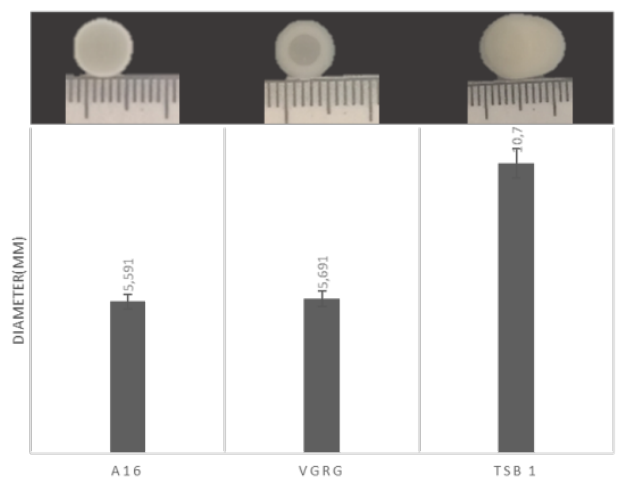
culture was spotted on $0.7 \%$ TY agar during 20 days. Values are the average of 2 assays (5 replicates/assay). 


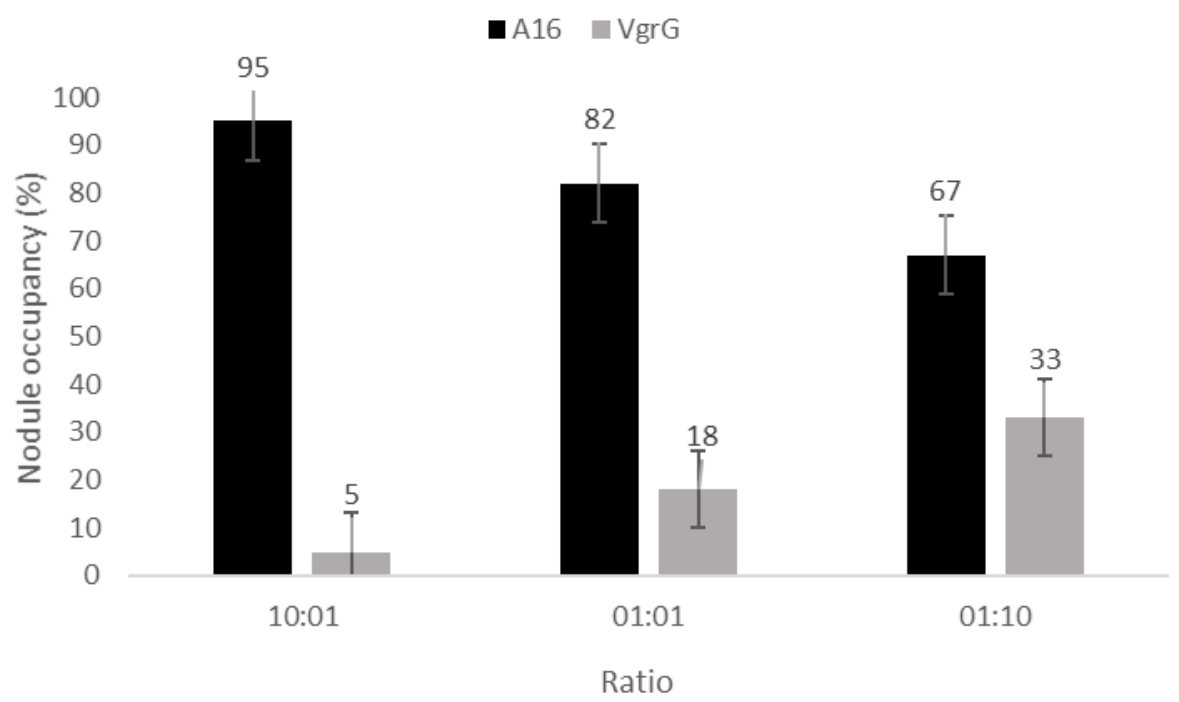

691 Fig. 6 Study of the competitiveness of the wild type A16 strain with the vgrG mutant at different ratios inoculated with $L$. angustifolius. Values are the average of at least 10 plants from 2 replicates (5 plants/replicate). 
Table S1. Bacterial strains and plasmids used in this work

\begin{tabular}{|c|c|c|}
\hline Strain or plasmids & Relevant characteristics & Reference of source \\
\hline \multicolumn{3}{|l|}{ E. coli strains } \\
\hline DH5 $\alpha$ & Host for DNA cloning & Hanahan 1983 \\
\hline S17.1 & Host for conjugation & Simon, Priefer and Puhler1983 \\
\hline \multicolumn{3}{|l|}{ Bradyrhizobium strains } \\
\hline LmicA16 (A16) & $\begin{array}{l}\text { Wild-type strain isolated from } \\
\text { Lupinus micranthus }\end{array}$ & Bourebaba et al., 2016 \\
\hline $\operatorname{vgr} G$ & A16 vgrG mutant, $\mathrm{Km}^{\mathrm{r}}$ & This work \\
\hline $\operatorname{vgr} G(\operatorname{pvgr} G)$ & $\operatorname{vg} r G$ strain complemented by pvgrG & This work \\
\hline$t s b 1$ & A16 tsbl mutant, $\mathrm{Km}^{\mathrm{r}}$ & This work \\
\hline \multicolumn{3}{|l|}{ Plasmids } \\
\hline PCR2.1 TOPO & $\begin{array}{l}\text { Vector for cloning PCR products, } \\
\mathrm{Amp}^{\mathrm{r}}, \mathrm{Km}^{\mathrm{r}}\end{array}$ & Invitrogen \\
\hline pK18mobsacB & Suicide vector; $s a c B, \mathrm{Km}^{\mathrm{r}}$ & Schäfer et al. 1994 \\
\hline $\mathrm{pK} 18 v g r G$ & $\begin{array}{l}\text { pK18mobsacB with a } 388 \mathrm{bp} \text { internal } \\
\text { fragment of } v g r G \text { from BA16 strain }\end{array}$ & This work \\
\hline $\begin{array}{l}\text { pVO-npt2- cefo-npt2- } \\
\text { GFP (pVO) }\end{array}$ & Suicide vector; $\mathrm{Cefo}^{\mathrm{r}}, \mathrm{Km}^{\mathrm{r}}$ & Okazaki et al., 2016 \\
\hline pVOtsb1 & $\begin{array}{l}\mathrm{pVO} \text { with a } 284 \mathrm{bp} \text { internal fragment } \\
\text { of } t \text { sblfrom BA16 strain }\end{array}$ & This work \\
\hline pMP220 & Broad-host-range plasmid, $\mathrm{Tc}^{\mathrm{r}}$ & Spaink et al. 1987 \\
\hline P6VgrG & $\begin{array}{l}\text { pMP220 derivative containing the } \\
\text { P } 6 v g r G \text { in front of reporter gene } \\
\text { lac } Z\end{array}$ & This work \\
\hline P6TssA & $\begin{array}{l}\text { pMP220 derivative containing the } \\
\text { P6tss } A \text { in front of reporter gene lac } Z\end{array}$ & This work \\
\hline pvgrG & $\begin{array}{l}\text { pMP220 derivative containing the } \\
\operatorname{vgr} G \text { under P6 promoter }\end{array}$ & This work \\
\hline pHC60 & $\begin{array}{l}\text { Broad-host range vector carrying } \\
\text { GFP under control of a constitutive } \\
\text { lac promoter, } \mathrm{Tc}^{\mathrm{r}}\end{array}$ & Cheng and Walker, 1998 \\
\hline
\end{tabular}

\section{References}

Bourebaba Y, Durán D, Boulila F, et al (2016) Diversity of Bradyrhizobium strains nodulating Lupinus micranthus on both sides of the Western Mediterranean: Algeria and Spain. Syst Appl Microbiol. 39:266-274. doi:10.1016/j.syapm.2016.04.006

Cheng HP, Walker GC (1998) Succinoglycan is required for initiation and elongation of infection threads during nodulation of alfalfa by Rhizobium meliloti. J Bacteriol. 180:5183-91. doi: 10.1128/JB.180.19.5183-5191.1998.

Hanahan D (1983) Studies on transformation of Escherichia coli with plasmids. J Mol Biol. 166:557-580.

Okazaki S, Tittabutr P, Teulet A, et al (2016) Rhizobium-legume symbiosis in the absence of Nod factors: two possible scenarios with or without the T3SS. ISME J.;10(1):64-74. doi:10.1038/ismej.2015.103.

Schäfer A, Tauch A, Jäger W et al (1994) Small mobilizable multi-purpose cloning vectors derived from the Escherichia coli plasmids pK18 and pK19: selection of defined deletions in the chromosome of Corynebacterium glutamicum. Gene. 145:69-73. 
709 Simon R, Priefer U, Puhler A (1983) A broad host range mobilization system for in vivo genetic engineering:

710 transposon mutagenesis in Gram-negative bacteria. Bio/Technology.1:784-91.

711 Spaink HP, Okker RJ, Wijffelman CA et al (1987) Promoters in the nodulation region of the Rhizobium

712 leguminosarum Sym plasmid pRL1JI. Plant Mol Biol. 9:27-39.

713 
Table S2. Primers used in this work

715

\begin{tabular}{|c|c|c|c|}
\hline Primer & Sequence & Amplicon & Use \\
\hline MutvgrG.A16 Fw & GCTGCCGAAATAGACGTGTT & \multirow[t]{2}{*}{$496 \mathrm{pb}$} & \multirow{2}{*}{$\begin{array}{l}\text { Obtaining vgrG } \\
\text { mutant }\end{array}$} \\
\hline MutvgrG.A16 Rv & ACTCCAAATCGTCGCATAGC & & \\
\hline P6VgrG Fw & CCCAAGCTTCCGTTTGCGTTGACGACC & \multirow[t]{2}{*}{$2.445 \mathrm{pb}$} & \multirow{2}{*}{$\begin{array}{l}\operatorname{vgrG} \text { mutant } \\
\text { complementation }\end{array}$} \\
\hline P6VgrG Rv & CCCAAGCTTGTAGCGTGCCAGCGGATA & & \\
\hline P6.Fw & GTCTTGAGCTCGCAAAGGCG & \multirow[t]{4}{*}{$690 \mathrm{pb}$} & \multirow{2}{*}{$\begin{array}{l}\text { Obtaining P6. tssA } \\
\text { and } \\
\text { P6.vgrG }\end{array}$} \\
\hline P6.Rv & TCGAGATCGCGGTTCAGGAT & & \\
\hline pMP220.Fw & AGCTCCTGAAAATCTCGTCG & & \multirow{2}{*}{$\begin{array}{l}\text { Determination of } \\
\text { P6 orientation }\end{array}$} \\
\hline pMP220.Rv & AACGGCCTCACCCCAAAAAT & & \\
\hline Mutmethyl.A16 Fw & CGGACTCTCGAGCCCGAACCACTTGGCGGCAATC & \multirow[t]{2}{*}{$388 \mathrm{pb}$} & \multirow{2}{*}{$\begin{array}{l}\text { Obtaining tsb1 } \\
\text { Mutant }\end{array}$} \\
\hline Mutmethyl.A16 Rv & $\begin{array}{l}\text { GCTGACTCTAGAGAGTCCCTGGGGTGCCAAATTC } \\
\end{array}$ & & \\
\hline
\end{tabular}

